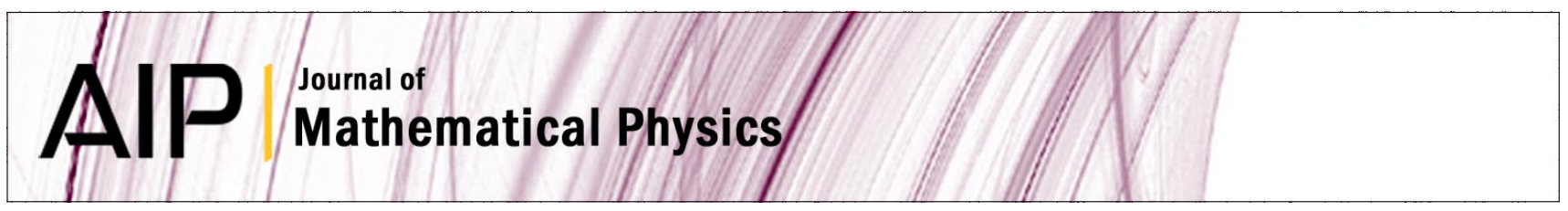

\title{
Remarks about inverse diffraction problem
}

\author{
A. G. Ramm
}

Citation: J. Math. Phys. 25, 2672 (1984); doi: 10.1063/1.526498

View online: http://dx.doi.org/10.1063/1.526498

View Table of Contents: http://jmp.aip.org/resource/1/JMAPAQ/v25/i9

Published by the American Institute of Physics.

\section{Additional information on J. Math. Phys.}

Journal Homepage: http://jmp.aip.org/

Journal Information: http://jmp.aip.org/about/about_the_journal

Top downloads: http://jmp.aip.org/features/most_downloaded

Information for Authors: http://jmp.aip.org/authors

\section{ADVERTISEMENT}

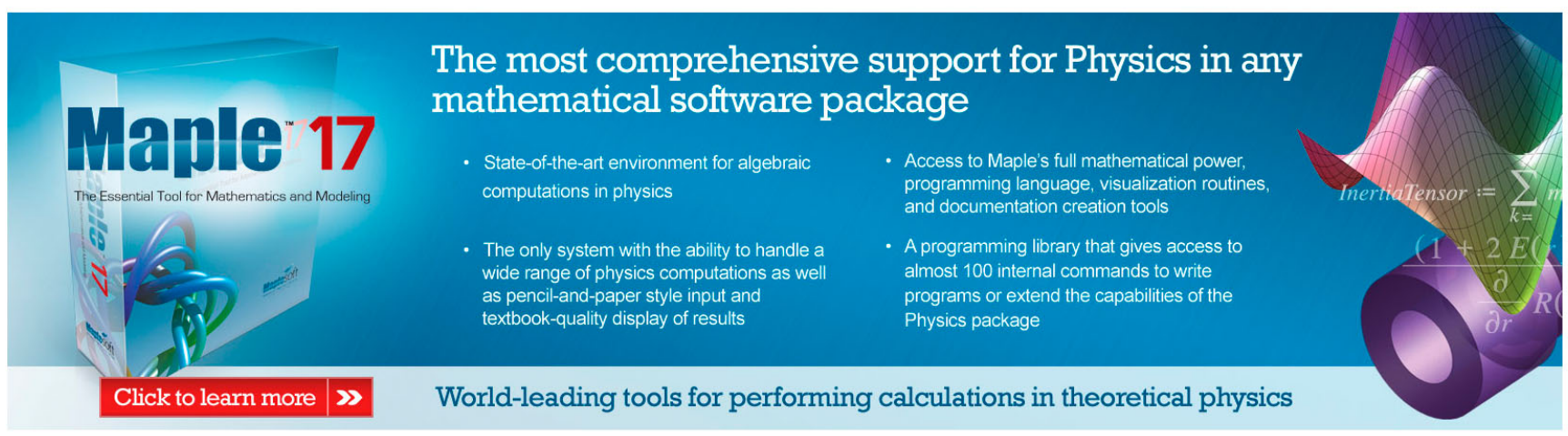




\title{
Remarks about inverse diffraction problem ${ }^{\text {a) }}$
}

\author{
A. G. Ramm \\ Mathematics Department, Kansas State University, Manhattan, Kansas 66506
}

(Received 10 August 1983; accepted for publication 20 January 1984)

From the scattering data one finds the support function or the principal curvatures of the surface of a reflecting obstacle. From either of these data the surface is effectively reconstructed.

PACS numbers: $03.80 .+r$

\section{INTRODUCTION}

Let $D$ be a bounded domain (an obstacle) with a smooth boundary $\Gamma$, and $\Omega=R^{3} \backslash D$ be the exterior domain. It is well known (see, e.g. Ref. 1) that the scattering amplitude $f$ for the problem

$$
\begin{aligned}
& \left(\nabla^{2}+k^{2}\right) u=0 \quad \text { in } \Gamma, \quad k>0, \\
& u=0 \text { on } \Gamma, \\
& u=\exp [i k(v, x)]+v, \\
& v \sim \frac{\exp (i k r)}{r} f(n, v, k) \quad \text { as }|x|=r \rightarrow \infty, \quad \frac{x}{r}=n,
\end{aligned}
$$

in the high-frequency $(k \rightarrow \infty)$ approximation determines the Gaussian curvature $K$ of $\Gamma$.

It was shown in Ref. 1 (see also Ref. 2) that $f$ determines the support function $a(l)$ of $\Gamma$ explicitly and the parametric equations of $\Gamma$ are

$$
x_{j}=-\frac{\partial a(l)}{\partial \alpha_{j}}, j=1,2,3 .
$$

Here $l=\left(\alpha_{1}, \alpha_{2}, \alpha_{3}\right)$ is a unit vector of normal to $\Gamma$ at the point $s_{0}$, which is uniquely determined by $v$ and $n$ if $\Gamma$ is strictly convex. Namely, $l=(n-v) /|n-v|$ and $s_{0}$ is the point at which the expression $(l, s)$ is stationary on $\Gamma_{+}$, where $\Gamma_{+}$is the illuminated part of $\Gamma$. The function $a(l)$ was defined in Ref. 2 as $a(l)=-\max _{s \in \Gamma_{+}}(l, s)$. The function $a(l)$ is a homogeneous function of $\alpha_{1}, \alpha_{2}, \alpha_{3}$ of order 1 :

$$
\frac{\partial a}{\partial \alpha_{j}} \alpha_{j}=a,
$$

one should sum over the repeated indices here and below. The basic result of Ref. 2 gives an algorithm for a stable calculation of $\Gamma$ from the knowledge of the scattering amplitude $f(n, v, k)$ for $n, v$ such that $(n-v) /|n-v|$ runs through all of the unit sphere $S^{2}$ (e.g., for $n=-v$ and $v$ runs through $S^{2}$, the backscattering case) and for two values of $k, k=k_{1}$, and $k_{2} \neq k_{1}$ such that the high-frequency asymptotic formula for $f$ holds $^{2}$ :

$$
\begin{gathered}
f_{j}=f\left(n, v, k_{j}\right)=-\frac{\exp \left[2 i k_{j}(n, l) a(l)\right]}{2\left[K\left(s_{0}\right)\right]^{1 / 2}}\left(1+\epsilon_{j}\right), \\
\epsilon_{j} \rightarrow 0 \text { as } k_{j} \rightarrow \infty .
\end{gathered}
$$

Here $K\left(s_{0}\right)$ is the Gaussian curvature of $\Gamma$ at the point $r_{0}$. If the $f_{j}$ are measured with the accuracy $\delta$, i.e., $\left|f_{j \delta}-f_{j}\right|<\delta$ and $f_{j \delta}$ are the measurements, and if $K\left(s_{0}\right) \leqslant d^{2}, d=$ const, then

\footnotetext{
a) Prepared while the author was visiting Schlumberger-Doll Research, $\mathbf{P}$ O. B. 307, Ridgefield, CT 06877.
}

$$
\begin{aligned}
\frac{f_{1 \delta}}{f_{2 \delta}}= & \exp \left[2 i(n, l) a(l)\left(k_{1}-k_{2}\right)\right] \\
& \times\left[1+O\left(\left|\epsilon_{1}\right|+\left|\epsilon_{2}\right|\right)+O(\delta d)\right] .
\end{aligned}
$$

Thus

$$
\begin{aligned}
a(l)= & \frac{1}{2 i(n, l)\left(k_{1}-k_{2}\right)} \ln \frac{f_{1 \delta}}{f_{2 \delta}} \\
& +O\left(\frac{\left|\epsilon_{1}\right|+\left|\epsilon_{2}\right|+\delta d}{\left|k_{1}-k_{2}\right||(n, l)|}\right) .
\end{aligned}
$$

The knowledge of $a(l)$ with a known error allows one to recover the surface stably using the following estimates of the derivatives of a function $a(l)$ known with an error

$$
\eta=o\left(\frac{\left|\epsilon_{1}\right|+\left|\epsilon_{2}\right|+\delta d}{\left|k_{1}-k_{2}\right||(n, l)|}\right) .
$$

Assume that $\left|a^{\prime \prime}\right| \leqslant M$, where $a^{\prime \prime}$ denotes any second derivative of $a\left(\alpha_{1}, \alpha_{2}, \alpha_{3}\right)$, and assume that $a_{\eta}$ is known, $\left|a_{\eta}-a\right|<\eta$. Let $h=(2 \eta / M)^{1 / 2}, \epsilon=\sqrt{2 M \eta}$. Then $\left|\left[a_{\eta}(\alpha+h b)-a_{\eta}(\alpha-h b)\right] / 2 h-\partial a / \partial b\right|<\epsilon=\sqrt{2 M \eta}$ (Ref. 3). Here $b$ is any unit vector, $\partial a / \partial b=\left(\nabla_{\alpha} a, b\right)$ is the derivative of $a$ in the direction $b$. All these facts are proved in Ref. 2 and provide a stable and effective algorithm for recovering the surface from the scattering data. One should be able to measure the phase of the scattered field in order to use the above algorithm. This is not very easy in practice. Therefore one can think of recovering the support function of $\Gamma$ from some other data.

It is clear from (7) that the Gaussian curvature $K=K_{1} K_{2}$, where the $K_{j}$ are the principal curvatures, can be determined from the measurements of $|f|^{2}$. Let us assume that the quantity $K_{1}-K_{2}$ can be measured. ${ }^{4}$ (In Ref. 4 a possibility to determine $K_{1}-K_{2}$ from the measurements of the scattered field was reported for electromagnetic scattering from a metallic body. It is not clear if $K_{1}-K_{2}$ can be found from the measurements of the scattered field in acoustic problems.) Then the quantity $h=K_{1}^{-1}+K_{2}^{-1}$ is known as a function of the unit normal $l=\left(\alpha_{1}, \alpha_{2}, \alpha_{3}\right)$ to $\Gamma$. From this data the support function of $\Gamma$ can be recovered and then the parametric equation of $\Gamma$ is given by (5). Notice, that if the origin of the coordinate system is placed inside the convex obstacle $D$, then $a(l)=\max _{\mathrm{se} \Gamma}(l, f)$, and $(5)$ takes the form

$$
x_{j}=\frac{\partial a}{\partial \alpha_{j}}, \quad 1 \leqslant j \leqslant 3
$$

\section{RECOVERING THE SURFACE FROM $h=K_{1}^{-1}+K_{2}^{-1}$}

In this section a construction of $\Gamma$ from $h$ is given. A similar construction can be found in the literature. ${ }^{5}$ Since it 
was not used in the inverse scattering problem, the construction is described in sufficient detail for convenience of the reader. It is worth mentioning that the problems of recovering a surface from its Gaussian curvature $K_{1} K_{2}$ or its mean curvature $\left(K_{1}+K_{2}\right) / 2$ were studied in the literature extensively. Both problems lead to nonlinear elliptic equations which cannot be solved explicitly. In contrast, the problem we are discussing here will lead to the Laplace equation which is effectively solvable:

$$
\frac{\partial^{2} a}{\partial \alpha_{1}^{2}}+\frac{\partial^{2} a}{\partial \alpha_{2}^{2}}+\frac{\partial^{2} a}{\partial \alpha_{3}^{2}} \equiv \nabla_{\alpha}^{2} a\left(\alpha_{1}, \alpha_{2}, \alpha_{3}\right)=h .
$$

To derive (9) one takes the Rodriguez formula

$$
d x_{j}-R d \alpha_{j}=0, \quad 1 \leqslant j \leqslant 3,
$$

in which $x=x\left(\alpha_{1}, \alpha_{2}, \alpha_{3}\right)$ is the radius vector of a point of the surface $\Gamma$, and the unit vector $l=\left(\alpha_{1}, \alpha_{2}, \alpha_{3}\right)$ serves as a parameter. There is a $1-1$ correspondence between the parameter $l=\left(\alpha_{1}, \alpha_{2}, \alpha_{3}\right)$ and the parameter $(\theta, \phi) \in S^{2}$. Namely, $l$ is determined by the point $(\theta, \phi) \in S^{2}$ and determines this point. Since $l$ is also the outer unit normal to $\Gamma$, we write $d \alpha_{j}$ in (10) instead of $-d N_{j}$ as in Ref. 5, where $N$ is the interior unit normal to $\Gamma$. Finally, $R$ in (10) is the radius of the curvature of the normal cut of $\Gamma$ in the principal direction $\left(d \alpha_{1}, d \alpha_{2}, d \alpha_{3}\right)$. From $\left(5^{\prime}\right)$ and $(10)$ it follows that

$$
\begin{aligned}
0 & =\frac{\partial^{2} a}{\partial \alpha_{j} \partial \alpha_{i}} d \alpha_{i}-R d \alpha_{j} \\
& =\left(\frac{\partial^{2} a}{\partial \alpha_{j} \partial \alpha_{i}}-R \delta_{j i}\right) d \alpha_{i}, \\
\delta_{j i} & =\left\{\begin{array}{l}
1, i=j, \\
0, i \neq j .
\end{array}\right.
\end{aligned}
$$

Since the vector $\left(d \alpha_{1}, d \alpha_{2}, d \alpha_{3}\right) \neq 0$ one concludes from (11) that

$$
\operatorname{det}\left(\frac{\partial^{2} a}{\partial \alpha_{j} \partial \alpha_{i}}-R \delta_{j i}\right)=0
$$

But det $\partial^{2} a / \partial \alpha_{j} \partial \alpha_{i}=0$. Indeed, differentiate (6) in $\alpha_{i}$ to obtain

$$
\frac{\partial^{2} a}{\partial \alpha_{j} \partial \alpha_{i}} \alpha_{j}+\frac{\partial a}{\partial \alpha_{j}} \delta_{i j}=\frac{\partial a}{\partial \alpha_{i}} \text { or } \frac{\partial^{2} a}{\partial \alpha_{j} \partial \alpha_{i}} \alpha_{j}=0
$$

Since $\left(\alpha_{1}, \alpha_{2}, \alpha_{3}\right) \neq 0$ one concludes from (13) that

$$
\operatorname{det} \frac{\partial^{2} a}{\partial \alpha_{j} \partial \alpha_{i}}=0
$$

From (14) it follows that Eq. (12) has a root $R=0$. Its two other roots are the principal radii $R_{j}=K_{j}^{-1}$, where $K_{j}$ are the principal curvatures. Since the body is assumed to be convex, $R_{j}>0$. The trace of the matrix $\partial^{2} a / \partial \alpha_{j} \partial \alpha_{i}$ is equal to $R_{1}+R_{2}+0$, where $R_{1}, R_{2}, 0$ are the roots of (12). Since $R_{1}+R_{2} \equiv h$ one obtains Eq. (9).

It remains to solve this equation. Let us expand $h=h\left(\alpha_{1}, \alpha_{2}, \alpha_{3}\right)=h(\theta, \phi)$ in spherical harmonics:

$$
h=\sum_{n>0} h_{n m} Y_{n m}(\theta, \phi),
$$

where the $Y_{n m}$ are the normalized in $L^{2}\left(S^{2}\right)$ spherical harmonics,

$$
\begin{aligned}
Y_{n m}= & \left(\frac{(2 n+1)(n-m) !}{4 \pi(n+m) !}\right)^{1 / 2} P_{n, m}(\cos \theta) e^{i m \phi}, \\
& -n \leqslant m \leqslant n,
\end{aligned}
$$

$P_{n, m}(x)$ are associated Legendre functions, and the $h_{n m}$ are the Fourier coefficients of $h$,

$$
h_{n m}=\int_{S^{2}} h(\theta, \phi) \overline{Y_{n m}} d \omega, \quad d \omega=\sin \theta d \theta d \phi .
$$

The function $a\left(\alpha_{1}, \alpha_{2}, \alpha_{3}\right)$ as a homogeneous function of order 1 can be expanded in the series $\left(A=a\left(r \alpha_{1}, r \alpha_{2}, r \alpha_{3}\right)\right.$

$\left.=\operatorname{ra}\left(\alpha_{1}, \alpha_{2}, \alpha_{3}\right)\right)$.

$$
A=r \sum_{n>0} A_{n m} Y_{n m},
$$

where $r>0$, and the function (17) is homogeneous of order 1 . The point $(r, \theta, \phi)$ corresponds to $\left(r \alpha_{1}, r \alpha_{2}, r \alpha_{3}\right)$.

Let us consider $(r, \theta, \phi)$ as spherical coordinates and $\left(y_{1}, y_{2}, y_{3}\right)$ as the corresponding Cartesian coordinates. Then $r=\left(\Sigma_{j=1}^{3} y_{j}^{2}\right)^{1 / 2}$,

$$
\begin{aligned}
\nabla_{y}^{2} r Y_{n m} & =\left(\frac{\partial^{2}}{\partial y_{1}^{2}}+\frac{\partial^{2}}{\partial y_{2}^{2}}+\frac{\partial^{2}}{\partial y_{3}^{2}}\right) r Y_{n m} \\
& =-\frac{(n-1)(n+2)}{r} Y_{n m},
\end{aligned}
$$

and from (17) and (18) it follows that

$$
\nabla_{y}^{2} A=-\sum_{n>0} A_{n m} \frac{(n-1)(n+2)}{r} Y_{n m} .
$$

Substitute (19) and (15) in (9), take $r=1$, and equate the coefficients in front of $Y_{n m}$ to obtain

$$
A_{n m}=-h_{n m} /(n-1)(n+2), \quad n \neq 1 .
$$

A necessary condition for a function $h(\theta, \phi)$ to be equal to $R_{1}+R_{2}$, where $R_{1}, R_{2}$ are the principal radii of a closed surface, is the equation

$$
h_{1 m}=0, \quad m=0, \pm 1 \text {. }
$$

This will be proved shortly. Assuming (21), one obtains an effective formula for the support function from (17) with $r=1$,

$$
a(l)=-\sum_{\substack{n>0 \\ n \neq 1}}^{\infty} \frac{h_{n m}}{(n-1)(n+2)} Y_{n m}(\theta, \phi) .
$$

Here $l$ is the unit vector corresponding to the point $(\theta, \phi) \in S^{2}$, and the $h_{n m}$ were defined in (16).

It remains to check (21). Let $d \sigma$ be the element of the surface area of $\Gamma, d \sigma=R_{1} d s_{1} R_{2} d s_{2}$, where $d s_{1} d s_{2}=d \omega$ is the element of the area of $S^{2},\left|R_{j} d s_{j}\right|, j=1,2$, are the elements of the length along the lines of principal curvatures on $\Gamma$, and $d s_{1} d s_{2}$ is the inner product of two vectors. Consider a family $\Gamma_{z}$ of parallel surfaces (for which the normals are the same as for $\left.\Gamma_{0}=\Gamma\right)$. Then

$$
\begin{aligned}
d \sigma_{z} & =\left(R_{1}+z\right) d s_{1}\left(R_{2}+z\right) d s_{2} \\
& =d \sigma+z\left(R_{1}+R_{2}\right) d \omega+z^{2} d \omega .
\end{aligned}
$$

From the Gauss formula it follows that

$$
\int_{\Gamma_{z}} N_{j} d \sigma_{z}=0
$$

where $N_{j}$ is the $j$ th component of the outer unit normal $N$ to $\Gamma_{z}, 0<z<z_{0}, z$ is arbitrary, and $z_{0}$ small, so that one can 
assume that $N$ does not depend on $z$. Substitute (23) in (24) to obtain

$$
\int_{S^{2}} h(l) N_{j} d \omega=\int_{S^{2}}\left(R_{1}+R_{2}\right) N_{j} d \omega=0, \quad 1 \leqslant j \leqslant 3 .(25)
$$

But $N_{j}=l_{j}$ are three linearly independent vectors in the space spanned by $Y_{1 m}, m=0, \pm 1$. Therefore (25) is equivalent to (21) because $h=R_{1}+R_{2}$ and $h_{1 m}$ is defined in (16). This completes the proof.

Example. If $\Gamma$ is a sphere, then $R_{1}=R_{2}=R=$ const, $h_{n m}=0$ for $n>0$. Therefore, $A_{n m}=0$ for $n>0$, $a(l)=A_{0} Y_{0}, Y_{0}=1 / \sqrt{4 \pi}$, by formula $(20), A_{0}=h_{0} / 2$, $h_{0}=2 R \sqrt{4 \pi}$. Thus $a(l)=R$ and one recovers $\Gamma$ from $h=2 R=$ const.

In the above construction it is not guaranteed that the support function $a(l)$ found from the given function $h(l)$, satisfying the necessary conditions (21), will correspond to a convex body $D$. In Ref. 6 a necessary and sufficient condition on $h$ for the corresponding $a(l)$ to be the support function of a convex body is given.

The main result from Ref. 6 for the three-dimensional case says: let $h(l)$ be a continuously differentiable function of the outer unit normal $l$ on the unit sphere $S^{2}$. For $h(l)$ to be the sum of the principal radii of curvature of a convex surface $\Gamma$ at the point at which the outer unit normal to $\Gamma$ is $l$, it is necessary and sufficient that the condition (21) and the following condition (26) hold:

$$
\int_{S^{2}} \frac{\left(l, l^{\prime \prime}\right)\left(\nabla h(l), l^{\prime \prime}\right)}{1-\left(l, l^{\prime}\right)} d \omega \leqslant 0,
$$

for all $l^{\prime}, l^{\prime \prime} \in S^{2}$ for which $\left(l^{\prime}, l^{\prime \prime}\right)=0$ with strict inequality in (26) for some choices of $l^{\prime}$ and $l^{\prime \prime}$. Here $\left(l, l^{\prime \prime}\right)$ is the inner product, and $\nabla h(l)$ is to be calculated as follows: define $h(l)$ for all $l \in R^{3}$ by the rule

$$
\begin{aligned}
& h(l)=\frac{1}{|l|} h\left(\frac{1}{|l|}\right), \quad|l|=\sqrt{\alpha_{1}^{2}+\alpha_{2}^{2}+\alpha_{3}^{2}}, \\
& l=\left(\alpha_{1}, \alpha_{2}, \alpha_{3}\right),
\end{aligned}
$$

calculate $\nabla h(l)$, and then set $|l|=1$.

The reason for extending $h$ as a positively homogeneous function of order -1 is that $a(l)$ is homogeneous of degree 1 , $\nabla^{2} a$ is homogeneous of degree -1 , and thus Eq. (9) holds for all $\left(\alpha_{1}, \alpha_{2}, \alpha_{3}\right) \in R^{3}$.

\section{ADDITIONAL GEOMETRICAL CONSIDERATIONS}

Let $x(l)$ be a vector function on $S^{2}$. It is called the normal representation of a convex nondegenerate body $D$ with the surface $\Gamma$ if $x(l)$ is that point of $\Gamma$ at which the outer normal to $\Gamma$ is $l$. If $\Gamma$ admits a tangent plane at any point then $x(l)$ is defined on all of $S^{2}$. Let us extend $x(l)$ from $S^{2}$ to $R^{3}$ by setting $x(u)=x(u /|u|)$, where $u \in R^{3},|u|=\left(u_{1}^{2}+u_{2}^{2}+u_{3}^{2}\right)^{1 / 2}, u \neq 0$. The value $x(0)$ does not play any role. The support function of $\Gamma$ is $(l, x(l))=a(l)$ provided that the origin is inside $D$. One has

$$
x_{j}=\frac{\partial a}{\partial \alpha_{j}}, \quad l=\left(\alpha_{1}, \alpha_{2}, \alpha_{3}\right), \quad 1 \leqslant j \leqslant 3,
$$

$$
\frac{\partial^{2} a}{\partial \alpha_{j} \partial \alpha_{i}}=\frac{\partial x_{i}}{\partial \alpha_{j}}=\frac{\partial x_{j}}{\partial \alpha_{i}},
$$

the matrix $a_{i j} \equiv \partial^{2} a / \partial \alpha_{i} \partial \alpha_{j}$ is semi-positive-definite. Since $a(\lambda l)=\lambda a(l), \lambda>0$, we define $a(0)=0$.

Differentiate Eq. (9) to obtain

$$
\nabla^{2} x_{j}(u)=\frac{\partial h}{\partial \alpha_{j}}
$$

where $h(u)=(1 /|u|) h(u /|u|)[$ see (27)].

In Ref. 6 the following proposition is proved: a continuously differentiable vector function $x(u)$ is a normal representation of a convex $\Gamma$ if and only if $\partial x_{i}(u) / \partial u_{j}$ is a semipositive-definite symmetric matrix not identically zero.

The necessity follows from (28), and $a(l)=(l, x(l))$ is the support function of a convex $\Gamma$. The sufficiency also follows from (28): construct $a(l)=(l, x(l))$, Eq. (28) shows that the Hessian $a_{i j}$ is a semi-positive-definite matrix. Since $x(u)$ is homogeneous of order 0 , one has $\left(u, \partial x(u) / \partial u_{j}\right)=0,1 \leqslant j \leqslant 3$. Therefore $a(u)$ is convex and, since $a(l)$ is convex and, since $a(l)$ is the support function of $\Gamma$, the surface $\Gamma$ is convex.

This condition can be formulated in terms of the coefficients $h_{n m}$ [see (15)] as semi-positive-definiteness of the matrix

$$
\begin{aligned}
a_{i j}= & -\sum_{\substack{n \geqslant 0 \\
n \neq 1}} \frac{h_{n m}}{(n-1)(n+2)} \\
& \times\left.\left(\frac{\partial^{2}}{\partial y_{i} \partial y_{j}} r Y_{n m}(\theta, \phi)\right)\right|_{r=1} \geqslant 0, \quad \forall(\theta, \phi) \in S^{2},
\end{aligned}
$$

where $a_{i j} \geqslant 0$ means that $a_{i j} t_{i} t_{j} \geqslant 0, \forall t_{i}$, and $y_{j}$ are the same as in formula (18).

Note added in proof: In Ref. 10 it was shown that $a(l)$ can be recovered stably from the knowledge of the scattering amplitude $f\left(n, v, K_{1}\right)$ at one high frequency and $n, v \in S^{2}$ such that $l=(n-v) /|n-v|$ runs through all of $S^{2}$. In Ref. 11 a uniqueness theorem for inverse diffraction problems with two-dimensional data is given.

\section{ACKNOWLEDGMENT}

The author thanks Professor K. Leichtweiss from the University of Stuttgart for a discussion and pointing out

Refs. 5-9.

${ }^{1}$ A. G. Ramm, Theory and Applications of Some New Classes of Integral
Equations (Springer, New York, 1980).
${ }^{2}$ A. G. Ramm, J. Math. Anal. Appl. (to appear, 1984).
${ }^{3}$ A. G. Ramm, Math. Meth. Appl. Sci. 3, $336(1981)$.
${ }^{4}$ S. Chaudhuri, W. Boerner, and B. Foo, "A High Frequency Inverse Scat-
tering Model to Recover the Specular Point Curvature from Parametric
Scattering Data," Department of Electrical Engineering, University of
Illinois, Chicago, preprint, 1983.
${ }^{5}$ W. Blaschke, Vorlesungen uber Differential Geometrie (Springer-Verlag,
Berlin, 1945), Vol. 1, p. 204.
${ }^{6}$ W. Firey, Mathematika 14, 1 (1967).
${ }^{7}$ A. Hurwitz, Ann. Ecol. Norm. 19, 357 (1902).
${ }^{8}$ E. Christoffel, J. Reine Angew. Math. 64, 193 (1865).
${ }^{9}$ C. Hsuing, Math. Z. 64, 41 (1956).
${ }^{10}$ A. G. Ramm, “Inverse diffraction problem," in Proceedings of NATO-
AR W-IMEI-1983 (D. Reidel, Dordrecht, 1984).
${ }^{11}$ A. G. Ramm, Phys. Rev. Lett. 52, 13 (1984); Phys. Lett. (to appear, 1984). 